\title{
Special theme issue: mechanisms of anesthesia
}

\author{
Donald R. Miller, MD • Sheldon H. Roth, PhD
}

Published online: 3 December 2010

(C) Canadian Anesthesiologists' Society 2010

Reflect for a moment on a relatively routine operating room scenario. First, standard anesthesia monitors are applied. Next, pre-oxygenation takes place, followed in sequence by the administration of fentanyl $100 \mu \mathrm{g} i v$, lidocaine $40 \mathrm{mg} i v$, propofol $180 \mathrm{mg} i v$, and rocuronium $50 \mathrm{mg} i v$. Then, the adult patient is rendered unconscious, and the patient's lungs are ventilated manually by bag-mask ventilation. This is followed by tracheal intubation and verification of end-tidal $\mathrm{CO}_{2}$ with the desflurane vaporizer opened to an initial dial concentration of $6 \%$ in oxygen. In the hands of a skilled and trained anesthesiologist, this example of yet another smooth induction is achieved with confidence and with a remarkable degree of predictability and patient safety.

The above sequence describes a surgical patient's rather straightforward but pharmacologically complex and remarkably dynamic transition from the awake state to a state of unconsciousness and paralysis with carefully attenuated autonomic responsiveness. Furthermore, this entire process is achieved simply within just a few minutes. This sequence occurs in our operating rooms many thousands of times each day worldwide. Nevertheless, how much do we truly understand the complex interactions of

D. R. Miller, MD ( $₫)$

Department of Anesthesia, The Ottawa Hospital, General Campus CCW, Room 1409, 501 Smyth Road, Ottawa, ON K1H 8L6, Canada

e-mail: dmiller@ottawahospital.on.ca

\section{S. H. Roth, PhD}

Department of Physiology \& Pharmacology, Hotchkiss Brain Institute, University of Calgary, Calgary, AB, Canada

\section{S. H. Roth, PhD}

Department of Anesthesiology, Hotchkiss Brain Institute, University of Calgary, Calgary, AB, Canada anesthetic drugs at their molecular sites of action? How exactly will furthering this understanding guide us towards developing even better anesthetics and designer drugs with enhanced predictability, broader pharmacodynamic responsiveness, and more favourable side effect profiles? After all, lest we as clinicians risk becoming pharmacologically complacent following a number of highly successful drug launches in the 1980s and 1990s, we realize with considerable concern that there have been relatively few major additions to our general anesthetic pharmacological armamentarium for over a decade.

Along these lines of concern, we embark upon a unique step for the Journal by devoting an entire issue to a single theme: mechanisms of anesthesia. The idea for the editorial content originates from the Eighth International Mechanisms of Anesthesia Conference (MAC) that was held at the University of Toronto in June 2010 (http://www.mac2010. org/).

The history and context of that meeting are described in an accompanying editorial. ${ }^{1}$ Since this conference capitalized on the participation and expertise of a large group of pre-eminent and world-renowned international researchers and clinical experts who presented a selection of their latest findings on the subject of mechanisms of anesthesia, we identified a unique opportunity to collate and publish selected elements of the meeting's scientific highlights for the benefit of our readers. Herein, we are pleased to present a series of brief reviews on highly innovative research regarding important aspects of anesthetic mechanisms and anesthetic pharmacology. Each of these reviews presents a critical and focused summary of a given topic on anesthetic mechanisms, and clinical implications are addressed to the greatest extent possible.

To begin, it is remarkable to consider that the mechanisms by which general anesthetics induce reversible loss 
of consciousness remain only partially understood. In their article ${ }^{2}$ Drs. Franks and Zecharia examine the evidence that anesthetic-induced loss of consciousness may be caused by actions on the neuronal pathways that produce natural sleep. Many general anesthetics produce electroencephalographic effects in the brain that are similar to the effects seen during non-rapid eye movement (non-REM) sleep. Gamma aminobutyric acid (GABA)ergic hypnogenic neurons are thought to be critical for generating non-REM sleep through their inhibitory projections to wake-active regions of the brain. Furthermore, the postsynaptic $\mathrm{GABA}_{\mathrm{A}}$ receptor, which is considered to be a major molecular target of many anesthetics, may be a point of convergence between natural sleep and general anesthesia. Indeed, there is growing evidence to show that modulating the release of neurotransmitters involved in the wake-active processes can impact anesthesia, supporting the concept that this point of convergence occurs at the level of the brain arousal systems. While general anesthetics can affect various points in the sleep-wake circuitry, the points that are true anesthetic targets remain to be seen. Sophisticated experimental approaches are necessary to address basic mechanisms of sleep and anesthesia and thus to advance our understanding in both of these fields.

As clinicians, we have recognized increasingly that development of the acute confusional state of delirium is associated with prolonged intensive care unit (ICU) and hospital length of stay, increased risk of functional decline, and higher mortality. Furthermore, disruption of sleep is a modifiable risk factor that may contribute to delirium and cognitive dysfunction in ICU patients, and disruption of sleep processes may produce acute confusion. Delirium develops through a complex interaction between the patient's predisposing risk factors and potentially modifiable events that occur during hospitalization, including sleep disruption and sedation. In their article, ${ }^{3}$ Drs. Sanders and Maze review the mechanisms of sedative-hypnotic action with respect to the risk of delirium imparted by drugs that act on $\mathrm{GABA}_{\mathrm{A}}$ receptors or $\alpha 2$ adrenoceptors. They hypothesize that these two receptor systems are causally linked through effects on memory. Their work helps us to understand why patients who receive an $\alpha 2$ adrenoceptor agonist are less likely to develop delirium (and the attendant cognitive dysfunction) compared with patients who receive benzodiazepines. The focus of future studies should be on a possible causal relationship between sedative administration, sleep disruption, and delirium.

While intraoperative amnesia is a desired endpoint of general anesthesia, we also recognize that intraoperative awareness with recall can result when learning and memory occur in a seemingly anesthetized patient. Anesthetics may also induce a state of "conscious amnesia" that can provide mechanistic insights into brain function with potential clinical applications. The hippocampal formation occupies a central position for the processing of sensory input into learned, remembered, and consciously retrievable information. In their article, ${ }^{4}$ Drs. Perouansky and Pearce review the current understanding of the role of the hippocampal formation in the generation of memory traces and how anesthetics might interfere with its function. Suppression of explicit memory is a fundamental component of general anesthesia. The authors review the mechanisms of memory formation and its perturbation by anesthetics with a focus on the network level.

The current generation of general anesthetics was developed more or less empirically, according to a given drug's ability to induce memory blockade, analgesia, immobility, and unconsciousness. However, at the molecular level, the exact mechanisms by which anesthetics produce their desirable behavioural endpoints and adverse side effects has remained elusive. Memory blockade is of particular interest to researchers and clinicians alike, because it is an essential component of the anesthetic state and occurs at drug doses considerably lower than those associated with sedation and immobility. In their review, ${ }^{5}$ Drs. Wang and Orser provide us with an overview of the acute memory-blocking properties of general anesthetics and the neuronal substrates that most likely contribute to memory loss. The cellular substrates of memory blockade include an increase in neuronal inhibition by $\mathrm{GABA}_{\mathrm{A}}$ receptors, a decrease in excitatory glutamatergic neurotransmission, and alterations in synaptic plasticity. Knowledge regarding the molecular basis of memory blockade may help to address memory disorders associated with anesthetic states, such as intraoperative awareness and memory deficits in the postoperative period.

It is now generally accepted that proteins are the primary targets of general anesthetics. However, showing that the activity of a protein is altered by general anesthetics at clinically relevant concentrations in vitro does not provide direct evidence that this target mediates pharmacological actions of general anesthetics. In their article, ${ }^{6}$ Dr. Drexler et al. use molecular mouse genetics to report on advances that have been made in identifying the contribution of individual ligand-gated ion channels to defined anesthetic endpoints. In particular, $\mathrm{GABA}_{\mathrm{A}}$ receptor subtypes defined by the presence of the $\alpha 1, \alpha 4, \alpha 5, \beta 2$, and $\beta 3$ subunits and the so-called "two-pore domain potassium channels" have been shown to have a mediating effect on the hypnotic, immobilizing, or amnesic actions of intravenous and volatile general anesthetics. Genetically modified mice have been very useful for research on mechanisms of anesthesia and have contributed to the functional identification of general anesthetic targets and to the role of these targets in neuronal networks.

Along the theme of allosteric binding sites and allosteric action, in their article, ${ }^{7}$ Drs. Forman and Miller identify the 
Cys-loop ligand-gated ion channel "superfamily" as a major group of neurotransmitter-activated receptors in the central and peripheral nervous system. The superfamily includes inhibitory receptors stimulated by GABA and glycine and excitatory receptors stimulated by acetylcholine and serotonin. This article presents current evidence on the location of the anesthetic binding sites on these channels and the mechanism by which binding to these sites alters their function. The second part of their review addresses the basis for this selectivity, while the third part describes the predictive power of a quantitative allosteric model of etomidate's actions on $\mathrm{GABA}_{\mathrm{A}}$ receptors. There is evidence that general anesthetics at clinical concentrations inhibit the excitatory receptors and enhance the inhibitory receptors. As explained by Drs. Forman and Miller, the location of general anesthetic binding sites on these receptors is being defined by photoactivable analogs of general anesthetics. The muscle-type nicotinic acetylcholine receptor has been studied the most extensively, and progress is now being made with $\mathrm{GABA}_{\mathrm{A}}$ receptors as well. Combining photolabelling with structure-function mutational studies in the context of allosteric mechanisms should lead to a more detailed understanding of how and where these important drugs act.

A major target action of general anesthetics is positive modulation of $\mathrm{GABA}_{\mathrm{A}}$ receptor-mediated inhibitory transmission. Using $\mathrm{R}(+)[3 \mathrm{H}]$ azietomidate, a photo incorporable analogue of the general anesthetic, $\mathrm{R}(+)$ etomidate, Drs. Olson and $\mathrm{Li}$ describe two amino acids that were affinity labelled in the M1 domain of the $\alpha$ subunit and in the M2 domain of the $\beta$ subunit of bovine GABAR. ${ }^{8}$ Complex laboratory modelling reveals that these two residues are adjacent on the transmembrane helices, forming a single type of binding pocket for the allosteric ligand in aqueous space behind the chloride channel. Etomidate labelling in the presence of other chemical classes of general anesthetics reveals that neurosteroids allosterically enhance binding of azietomidate, while isoflurane completely and possibly competitively blocks such binding. Propofol and barbiturates stereospecifically inhibit etomidate binding, but only partially, probably due to allosteric interaction. The alcohols, n-octanol and ethanol, at relevant concentrations fail to affect etomidate binding. Thus, this etomidate binding pocket at the $\beta / \alpha$ interface in the transmembrane domain sits directly below the GABA binding site at the same interface in the extracellular domain and plays a role in the modulation of $\mathrm{GABA}_{\mathrm{A}}$ receptor function by those anesthetic classes that affect etomidate binding. The foregoing are several examples of novel insights into specific sites of drug interaction within the $\mathrm{GABA}_{\mathrm{A}}$ receptor.

Finally, a growing body of evidence suggests that general anesthetics may be neurotoxic to both the aging and young brain. Postoperative cognitive decline in the elderly has emerged as a major health concern, and there is a growing interest in the potential relationship between general anesthetic exposure and the onset and progression of Alzheimer's disease. Despite the animal data suggesting a relationship between anesthesia and neurotoxicity in the elderly, a definitive link remains elusive in humans. The available evidence of a possible association between anesthesia, surgery, and long-term cognitive effects, including Alzheimer's disease, deserves consideration. In their review, ${ }^{9}$ Dr. Bittner et al. summarize the evidence for anesthesia-induced neurotoxicity in the elderly, while highlighting the limitations of existing data in a clinical context.

It is our sincere hope that practicing anesthesiologists, clinician researchers, and basic scientists alike will appreciate this collection of reviews and important updates that reflect certain aspects of the current state of knowledge relating to anesthetic mechanisms. Pharmacological innovation depends on the body of scientific knowledge that drives the process. Translation of this knowledge is critical for advancing clinical care in both healthy and medically complex patients; namely, the millions of patients worldwide who receive general anesthetics each year.

\section{Numéro spécial: les mécanismes de l'anesthésie}

Réfléchissons un instant à une situation relativement courante en salle d'opération. Tout d'abord, on met en place les moniteurs habituels pour l'anesthésie. Ensuite il y a pré-oxygénation, laquelle est suivie en séquence par l'administration de fentanyl $100 \mu \mathrm{g} i v$, de lidocaïne $40 \mathrm{mg}$ $i v$, de propofol $180 \mathrm{mg} i v$, et de rocuronium $50 \mathrm{mg} i v$. Le patient, un adulte, est ainsi rendu inconscient, et ses poumons sont ventilés manuellement à l'aide d'un ballon. Une fois cette étape accomplie, l'intubation endotrachéale a lieu et le $\mathrm{CO}_{2}$ télé-expiratoire est vérifié; le vaporisateur de desflurane est ouvert à une concentration initiale de $6 \%$ avec de l'oxygène. Dans les mains d'un anesthésiologiste compétent et formé, cet exemple d'une induction sans complication se fait aisément et possède un degré de prévisibilité impressionnant - sans oublier une sécurité incontestée pour le patient.

La séquence ci-dessus décrit la transition relativement simple - tout en étant étonnamment dynamique et complexe d'un point de vue pharmacologique - de l'état d'éveil à un état d'inconscience et de curarisation, avec une réaction autonome dont l'ampleur a été atténuée avec soin. De plus, ce processus s'accomplit entièrement en l'espace de quelques minutes seulement. Cette séquence se déroule des milliers de fois chaque jour dans nos salles d'opération 
à travers le monde. Cependant, une question se pose: que comprenons-nous vraiment des interactions complexes des médicaments anesthésiques à leurs sites d'action moléculaires? Comment une meilleure compréhension de ces mécanismes nous guidera-t-elle exactement vers la mise au point d'anesthésiques et de médicaments sur mesure encore plus performants et bénéficiant d'une prévisibilité améliorée, d'une sensibilité pharmacodynamique plus étendue et de profils d'effets secondaires plus favorables? Après tout, au cas où nous, en tant que cliniciens, devenions trop confiants en notre arsenal pharmacologique à la suite de plusieurs lancements très réussis de médicaments dans les années 1980 et 1990, nous prenons conscience, non sans inquiétude, qu'il n'existe que relativement peu d'ajouts majeurs à notre arsenal pharmacologique en anesthésie générale depuis plus de dix ans.

Dans le but d'aborder ces préoccupations, nous franchissons une étape distinctive en consacrant un numéro entier du Journal à un seul thème: les mécanismes de l'anesthésie. L'idée du contenu éditorial a vu le jour lors de la $8^{\mathrm{e}}$ Conférence internationale sur les mécanismes de l'anesthésie (MAC) qui s'est tenue en juin 2010 à l'Université de Toronto (http://www.mac2010.org/).

L'histoire de cette conférence et le contexte dans lequel elle s'est déroulée sont décrits plus en détail dans un autre éditorial. ${ }^{1}$ Comme cette conférence s'est appuyée sur la participation et l'expertise d'un groupe important de chercheurs et d'experts cliniciens de renommée internationale, lesquels ont présenté quelques-unes de leurs dernières découvertes sur le thème des mécanismes de l'anesthésie, nous avons saisi cette occasion unique pour colliger et publier certains éléments parmi les points forts de la conférence au profit de nos lecteurs. Ainsi, nous avons le plaisir de présenter une série d'articles de synthèse courts décrivant des recherches très innovantes portant sur divers aspects importants des mécanismes de l'anesthésie et de la pharmacologie des agents anesthésiants. Chacun de ces articles comprend un résumé cohérent et ciblé sur un sujet donné traitant des mécanismes de l'anesthésie, et les implications cliniques sont abordées autant que possible.

Tout d'abord, il est tout à fait remarquable de penser que les mécanismes par lesquels les anesthésiques généraux provoquent une perte de conscience réversible ne sont que partiellement connus. Dans leur article, ${ }^{2}$ les Drs Franks et Zecharia examinent les données probantes qui suggèrent que la perte de conscience induite par anesthésie est provoquée par des actions sur les voies neuronales qui produisent le sommeil normal. De nombreux anesthésiques généraux provoquent des effets électroencéphalographiques au niveau du cerveau, lesquels sont semblables aux effets observés pendant la phase lente (non paradoxale) du sommeil. On pense que les neurones hypnotiques gabaergiques sont des éléments cruciaux pour la génération du sommeil lent en raison de leurs projections inhibitrices aux régions du cerveau actives à l'éveil. En outre, le récepteur $\mathrm{GABA}_{\mathrm{A}}$ post-synaptique est une cible moléculaire importante de plusieurs anesthésiques et pourrait par conséquent constituer un point de convergence entre le sommeil normal et l'anesthésie générale. En effet, un nombre croissant de données probantes suggèrent que la modulation de la libération des neurotransmetteurs impliqués dans les processus actifs pendant l'éveil pourrait avoir un impact sur l'anesthésie, ce qui appuie l'hypothèse que ce point de convergence survient au niveau des systèmes de réveil du cerveau. Alors que les anesthésiques généraux peuvent avoir des effets sur divers points du circuit veille-sommeil, les points constituant de véritables cibles anesthésiques doivent encore être déterminés. Il faudra utiliser des approches expérimentales perfectionnées pour aborder les mécanismes de base du sommeil et de l'anesthésie. De telles approches devraient faire progresser notre compréhension dans ces deux domaines.

En tant que cliniciens, nous sommes de plus en plus conscients que l'apparition d'une confusion mentale et de delirium est associée à une durée de séjour prolongée à l'unité des soins intensifs (USI) et à l'hôpital, à un risque accru de déclin fonctionnel, et à une mortalité plus élevée. En outre, la perturbation du sommeil est un facteur de risque modifiable qui pourrait contribuer au delirium et à la dysfonction cognitive chez les patients de l'USI, et la perturbation des processus du sommeil pourrait provoquer une confusion mentale aiguë. Le delirium se manifeste en raison d'une interaction complexe entre les facteurs de risque prédisposant le patient et des événements potentiellement modifiables qui surviennent pendant l'hospitalisation, notamment les perturbations du sommeil et la sédation. Dans leur article, ${ }^{3}$ les Drs Sanders et Maze passent en revue les mécanismes d'action des sédatifs-hypnotiques par rapport au risque de delirium imparti par les médicaments qui agissent sur les récepteurs de l'acide $\gamma$-amino-butyrique de type $\mathrm{A}\left(\mathrm{GABA}_{\mathrm{A}}\right)$ et les adrénocepteurs $\alpha 2$. Leur hypothèse est que ces deux systèmes de récepteurs entretiennent un lien causal par le biais d'effets au niveau de la mémoire. Leurs travaux nous aident à comprendre pourquoi les patients recevant un agoniste des adrénorécepteurs $\alpha 2$ sont moins enclins à manifester un delirium (et la dysfonction cognitive concomitante) que les patients recevant des benzodiazépines. Les études futures devraient essayer de déterminer s'il existe une relation de causalité entre l'administration de sédatifs, les perturbations du sommeil et le delirium.

Bien que l'amnésie peropératoire soit un résultat désirable de l'anesthésie générale, nous sommes également conscients qu'il peut y avoir conscience peropératoire avec souvenir lorsqu'il y a apprentissage et mémorisation chez 
un patient apparemment anesthésié. Les agents anesthésiques peuvent également induire un état d'amnésie consciente, qui peut nous donner des perspectives quant aux mécanismes de la fonction cérébrale, lesquels pourraient avoir des applications cliniques. L'hippocampe joue un rôle central dans le traitement et la transformation des informations sensorielles en informations apprises, remémorées et consciemment récupérables. Dans leur article, ${ }^{4}$ les Drs Perouansky et Pearce passent en revue comment on voit actuellement le rôle de l'hippocampe dans la génération d'engrammes et de la façon dont les agents anesthésiques pourraient empêcher son fonctionnement. La suppression de la mémoire explicite est une composante essentielle de l'anesthésie générale. Les auteurs passent en revue les mécanismes de formation de la mémoire et de son dysfonctionnement par les anesthésiques notamment par leurs effets sur les réseaux neuronaux.

La génération actuelle d'anesthésiques généraux a été mise au point de façon plus ou moins empirique, selon la capacité d'un médicament donné à à provoquer l'amnésie, l'analgésie, l'immobilité et l'inconscience. Toutefois, au niveau moléculaire, les mécanismes précis qui permettent aux anesthésiques de produire leurs effets désirés sur le comportement et les effets secondaires défavorables sont encore peu connus. L'amnésie est particulièrement intéressante pour les chercheurs et les cliniciens, car il s'agit d'une composante essentielle de l'état anesthésié et survient à des doses de médicament significativement plus basses que celles associées à la sédation ou à l'immobilité. Dans leur compte-rendu, ${ }^{5}$ les Drs Wang et Orser présentent brièvement les propriétés amnésiantes aiguës des anesthésiques généraux ainsi que les substrats neuronaux qui contribuent très probablement à la perte de mémoire. Les substrats cellulaires de l'amnésie comprennent une augmentation de l'inhibition neuronale des récepteurs $\mathrm{GABA}_{\mathrm{A}}$, une diminution de la neurotransmission excitatoire glutamatergique, et des modifications au niveau de la plasticité synaptique. Les connaissances concernant la base moléculaire du blocage de la mémoire pourraient nous permettre de mieux comprendre et traiter les troubles de la mémoire associés à l'état anesthésié, tels que la conscience peropératoire et les troubles de mémoires en période postopératoire.

Aujourd'hui, il est universellement accepté que les protéines sont les cibles principales des anesthésiques généraux. Cependant, la démonstration que l'activité d'une protéine est modifiée in vitro par les anesthésiques généraux à des concentrations pertinentes d'un point de vue clinique ne constitue pas une donnée probante directe prouvant que cette cible est le médiateur des effets pharmacologiques des anesthésiques généraux. Dans leur article, ${ }^{6}$ le Dr Drexler et coll. rapportent les progrès accomplis, en utilisant la génétique moléculaire chez la souris, dans la détermination du rôle de plusieurs canaux ioniques sensibles à un ligand quant à l'obtention de critères reliés à l'anesthésie. Plus particulièrement, on a découvert que les sous-types de récepteurs $\mathrm{GABA}_{\mathrm{A}}$ tels que définis par la présence des sous-unités $\alpha 1, \alpha 4, \alpha 5, \beta 2$, et $\beta 3$ et les canaux potassiques à deux domaines $\mathrm{P}$ exerçaient un effet médiateur sur les actions hypnotiques, immobilisantes ou amnésiques des anesthésiques généraux intraveineux ou volatils. Les souris génétiquement modifiées ont été très utiles à la recherche sur les mécanismes de l'anesthésie et ont contribué à l'identification fonctionnelle des cibles des anesthésiques généraux et du rôle de ces cibles dans les réseaux neuronaux.

Dans leur article, ${ }^{7}$ les Drs Forman et Miller se sont intéressés aux sites de liaison allostérique et à l'effet allostérique; ils ont identifié la 'super-famille' des canaux ioniques sensibles à un ligand de la boucle Cys en tant que groupe majeur de récepteurs activés par les neurotransmetteurs dans les systèmes nerveux central et périphérique. Cette superfamille comprend des récepteurs inhibiteurs stimulés par la GABA et la glycine ainsi que des récepteurs excitateurs stimulés par l'acétylcholine et la sérotonine. Ce compte-rendu présente les données probantes actuelles sur l'emplacement des sites de liaison des anesthésiques sur ces canaux et le mécanisme par lequel la liaison sur ces sites en particulier modifie leur fonctionnement. La deuxième partie de leur article traite des bases de cette sélectivité, et la troisième partie décrit le pouvoir de prédiction d'un modèle allostérique quantitatif montrant l'effet de l'étomidate sur les récepteurs $\mathrm{GABA}_{\mathrm{A}}$. Certaines données probantes suggèrent que les anesthésiques généraux administrés à des concentrations habituelles en clinique inhibent les récepteurs excitateurs et stimulent les récepteurs inhibiteurs. Comme l'expliquent les Drs Forman et Miller, l'emplacement des sites de liaison des anesthésiques généraux sur ces récepteurs est défini par des analogues photoactivés des anesthésiques généraux. Le récepteur qui a été le mieux étudié est le récepteur nicotinique de l'acétylcholine à la plaque motrice, et des progrès sont actuellement en cours au niveau de nos connaissances des récepteurs $\mathrm{GABA}_{\mathrm{A}}$. La combinaison du marquage par photoaffinité et d'études de mutation sur la fonction et la structure dans le contexte des mécanismes allostériques devrait nous permettre de mieux comprendre où ces importants médicaments agissent et comment ils le font.

Une cible d'action majeure des anesthésiques généraux est la modulation positive de la transmission inhibitrice médiée par les récepteurs $\mathrm{GABA}_{\mathrm{A}}$. À l'aide d'aziétomidate $\mathrm{R}(+)[3 \mathrm{H}]$, un analogue photo-incorporable de l'anesthésique général étomidate $\mathrm{R}(+)$, les Drs Olson et Li décrivent deux acides aminés marqués par affinité dans le domaine M1 de la sous-unité $\alpha$ et dans le domaine M2 de la 
sous-unité $\beta$ des R-GABA bovins. ${ }^{8}$ Des modèles de laboratoire complexes ont révélé que ces deux résidus sont adjacents sur les hélices transmembranaires, formant un type unique de poche de liaison pour le ligand allostérique dans l'espace aqueux derrière le canal chlore. Le marquage de l'étomidate en présence d'anesthésiques généraux appartenant à d'autres classes chimiques révèle que les neurostéroïdes augmentent la liaison de l'aziétomidate de façon allostérique, alors que l'isoflurane bloque complètement, peut-être de façon compétitive, une telle liaison. Le propofol et les barbituriques inhibent la liaison de l'étomidate de façon stéréospécifique, mais seulement partiellement, ce qui est probablement dû à une interaction allostérique. Les alcools n-octanol et éthanol, lorsqu'ils sont à des concentrations pertinentes, n'affectent pas la liaison de l'étomidate. Ainsi, cette poche de liaison de l'étomidate au niveau de l'interface $\beta / \alpha$ dans le domaine transmembranaire se situe directement au-dessous du site de liaison GABA à la même interface dans le domaine extracellulaire et joue un rôle dans la modulation de la fonction des récepteurs $\mathrm{GABA}_{\mathrm{A}}$ par les classes d'anesthésiques qui affectent la liaison de l'étomidate. Il s'agit là de plusieurs exemples de perspectives nouvelles concernant certains des sites spécifiques d'interaction médicamenteuse dans le récepteur $\mathrm{GABA}_{\mathrm{A}}$.

Enfin, un nombre croissant de données probantes suggèrent que les anesthésiques généraux pourraient avoir des effets neurotoxiques sur le cerveau, qu'il soit jeune ou âgé. Le déclin cognitif postopératoire chez le patient âgé est aujourd'hui un problème de santé publique majeur et un intérêt croissant est porté à la relation potentielle entre l'exposition à un anesthésique général et l'apparition et l'évolution de la maladie d'Alzheimer. Malgré les données animales suggérant une relation entre l'anesthésie et la neurotoxicité chez le sujet âgé, il est difficile d'établir un lien incontestable chez l'humain. Les données probantes disponibles portant sur une association possible entre l'anesthésie, la chirurgie, et des effets cognitifs à long terme, y compris la maladie d'Alzheimer, méritent d'être examinées. Dans leur compte-rendu, ${ }^{9}$ le Dr Bittner et coll. résument les données probantes rapportant des cas de neurotoxicité induite par l'anesthésie chez le patient âgé, tout en insistant sur les limites des données existantes dans un contexte clinique.

Nous espérons sincèrement que les anesthésiologistes en pratique, les chercheurs cliniciens et les chercheurs en science fondamentale apprécieront cette collection d'articles de synthèse et de mises à jour importantes. Ils reflètent certains aspects de l'état actuel de nos connaissances concernant les mécanismes de l'anesthésie. L'innovation pharmacologique dépend du corpus de connaissances scientifiques qui la stimulent. La traduction de ces connaissances est cruciale si l'on souhaite faire progresser les soins cliniques chez les patients sains et chez les patients plus complexes d'un point de vue médical - en d'autres termes, les millions de patients dans le monde qui reçoivent des anesthésiques généraux chaque année.

Competing interests There were no peer-reviewed grants or sources of commercial funding in support of this special theme issue. Competing interests for the authors of the individual review articles and brief reviews are identified within each article in this issue of the Journal. Dr. Roth was one of the organizers of MAC 2010.

\section{References}

1. Miller KW, Orser BA, Roth SH. Mechanisms of anesthesia: past, present, and a glimpse into the future. Can J Anesth 2011; 58. doi: 10.1007/s12630-010-9433-y.

2. Franks NP, Zecharia AY. Sleep and general anesthesia. Can $\mathbf{J}$ Anesth 2011; 58. doi:10.1007/s12630-010-9420-3.

3. Sanders RD, Maze M. Contribution of sedative-hypnotic agents to delirium via modulation of the sleep pathway. Can J Anesth 2011; 58. doi:10.1007/s12630-010-9421-2.

4. Perouansky M, Pearce RA. How we recall (or don't): the hippocampal memory machine and anesthetic amnesia. Can J Anesth 2011; 58. doi:10.1007/s12630-010-9417-y.

5. Wang $D$, Orser $B A$. Inhibition of learning and memory by general anesthesia. Can J Anesth 2011; 58. doi:10.1007/s12630-0109428-8.

6. Drexler B, Antkowiak B, Engin E, Rudolph U. Identification and characterization of anesthetic targets by mouse molecular genetics approaches. Can J Anesth 2011; 58. doi:10.1007/s12630-0109414-1.

7. Forman $S A$, Miller $K W$. Anesthetic sites and allosteric mechanisms of action on Cys-loop ligand-gated ion channels. Can J Anesth 2011; 58. doi:10.1007/s12630-010-9419-9.

8. Olsen $R W, L i G D$. $\mathrm{GABA}_{\mathrm{A}}$ receptors as molecular targets of anesthetics: identification of binding sites provides clues to allosteric modulation. Can J Anesth 2011; 58. doi:10.1007/s12630010-9429-7.

9. Bittner EA, Yиe Y, Xie Z. Brief review: anesthetic neurotoxicity in the elderly, cognitive dysfunction and Alzheimer's disease. Can J Anesth 2011; 58. doi:10.1007/s12630-010-9418-x. 